\title{
Endotracheal temperature and humidity measurements in laryngectomized patients: intra- and inter-patient variability
}

\author{
R. J. Scheenstra $\cdot$ S. H. Muller $\cdot$ A. Vincent $\cdot$ \\ M. Sinaasappel $\cdot$ J. K. Zuur $\cdot$ Frans J. M. Hilgers
}

Received: 21 January 2009/Accepted: 3 May 2009/Published online: 26 May 2009

(c) The Author(s) 2009. This article is published with open access at Springerlink.com

\begin{abstract}
This study assesses intra- and inter-patient variability in endotracheal climate (temperature and humidity) and effects of heat and moister exchangers (HME) in 16 laryngectomized individuals, measured repeatedly $(N=47)$. Inhalation Breath Length (IBL) was $1.35 \mathrm{~s}$ without HME and $1.05 \mathrm{~s}$ with $\operatorname{HME}(P<0.0001)$. With HME, end-inspiratory (minimum) humidity values increased $5.8 \mathrm{mg} \mathrm{H}_{2} \mathrm{O} / \mathrm{L} \quad(P<0.0001)$ and minimum temperature values decreased $1.6^{\circ} \mathrm{C}(P<0.0001)$. For the temperature and humidity minimums, the inter-patient variability was much smaller than the short- and long-term
\end{abstract}

\footnotetext{
R. J. Scheenstra · J. K. Zuur · F. J. M. Hilgers ( $\square)$

Department of Head and Neck Oncology and Surgery,

The Netherlands Cancer Institute,

Antoni van Leeuwenhoek Hospital,

Plesmanlaan 121, 1066 CX Amsterdam,

The Netherlands

e-mail: f.hilgers@nki.nl

R. J. Scheenstra · F. J. M. Hilgers

Department of Otorhinolaryngology, Academic Medical Centre,

University of Amsterdam, Amsterdam, The Netherlands

S. H. Muller - M. Sinaasappel

Department of Nuclear Medicine-Radiology,

The Netherlands Cancer Institute,

Antoni van Leeuwenhoek Hospital,

Amsterdam, The Netherlands

A. Vincent

Department of Biostatistics,

The Netherlands Cancer Institute,

Antoni van Leeuwenhoek Hospital, Amsterdam,

The Netherlands

F. J. M. Hilgers

Institute of Phonetic Sciences/ACLC, University of Amsterdam,

Amsterdam, The Netherlands
}

intra-patient variability. For exhalation breath length and full breath length, the opposite was the case. Conclusions: (1) Because inter-patient variability is smaller than intrapatient variability, investigating endotracheal climate in a limited number of laryngectomized subjects is justified, provided repeated measurements per patient are accomplished; (2) main contributor to intra-patient variability is the positioning of the catheter tip in the trachea; (3) an HME leads to a shortened IBL which enhances the HME effect.

Keywords Total laryngectomy - Tracheal climate . Temperature and humidity

\section{Introduction}

Total laryngectomy causes a permanent disconnection of the upper and lower airways. Lack of conditioning of inspired air in these patients leads to an increase of chronic pulmonary complaints like frequent involuntary coughing, sputum production, and repeated daily forced expectoration in order to clear the airways [4]. Passive humidifiers (i.e. heat and moisture exchangers; HMEs) were developed to compensate for the lost upper airway function and have been found to reduce these symptoms and improve quality of life $[1,4,5]$.

The heat and moisture exchanging capacity of HMEs is widely proven in laboratory studies in vitro. Measurement of the endotracheal temperature and humidity in vivo is more complex since the tracheal mucosa obviously has 'HME-properties', resulting in measurements of two HME in series. In vivo temperature and humidity measurements are also technically very challenging since no commercial measurement system is available for this purpose. Therefore, it is not surprising that only a few studies exist on the 
endotracheal HME effect in laryngectomized patients, all of them based on a small number of patients [6-8, 11, 14].

To date no study has investigated patient variability in endotracheal temperature and humidity. It is not known whether similar results will be obtained if measurements are repeated within a limited time-period (short-term variation), or whether temperature and humidity values fluctuate over longer time periods due to, for example, changes in the condition of the mucosa (long-term variation). Also the impact of patient characteristics such as individual diversity in breathing patterns [2] is not known.

The primary aim of this study is to determine the intraindividual variability (and the contributions of both shortand long-term factors) and the inter-individual variability in endotracheal temperature and humidity in laryngectomized patients. In addition we investigate whether our previously reported HME effects on endotracheal temperature and humidity [14] can be reproduced if a larger number of repeated measurements is included.

\section{Patients and methods}

\subsection{Patients}

In this study we analyzed data pooled from three studies investigating different HME devices in the period between March 2007 and March 2008. These studies were performed at room conditions in the Netherlands Cancer Institute in Amsterdam and include 16 laryngectomized patients; 15 male and 1 female (median age 67 years; range 47-81 years, SD 9.1 years). All patients also had been treated with radiotherapy, had quit smoking and were in long-term follow-up, on average 8.0 years postoperative (median 6.0 years, range $0.6-19$ years, SD 5.8 years).

\subsection{Study design}

Due to the use of pooled data, the study design is rather complicated and is shown schematically in Fig. 1. In the 1 st and 3rd study period, several patients were measured on multiple days. Each measurement was repeated up to five times on the same day (repetitions) The time between 2 days within a study period was not longer than 22 days (mean 8.8 days).

\subsection{Measurement protocol}

All studies were accomplished according to the same measurement protocol, approved by the Protocol Review Board of the in the Netherlands Cancer Institute and written informed consent had been obtained from all patients.

During each measurement session the patient was seated in a chair and was asked to breath calmly. A small hole was punched in a peristomal HME adhesive (Provox, Atos Medical, Hörby, Sweden), through which the distal tip of the sample catheter of the airway climate explorer (ACE; described below) was inserted. The catheter tip was held approximately $1 \mathrm{~cm}$ behind the stoma opening in the trachea. Each measurement session included one $10 \mathrm{~min}$ breathing period without an HME (open stoma breathing) and at least one, but in most instances two $10 \mathrm{~min}$ breathing periods with an HME covering the stoma. The sequence of measurements with or without an HME was randomized in all instances. The 10 min breathing periods will be referred to as observations. Three different HMEs were assessed within the framework of this study. Although the differences between the various HMEs are considered as a confounder in this analysis, the humidity and temperature values for the Normal Provox HME (Atos Medical AB,
Fig. 1 The schematic overview of the study design is illustrated using the measurements of patient $\mathrm{nr} 1$ as an example (see also Table 1). In three different study periods repeated measurements are performed either on one day or on multiple days. One measurement consisted of several, usually three, observations, $10 \mathrm{~min}$ without HME, 10 min with one HME and 10 min with another HME in a randomized sequence. One observation had a $5 \mathrm{~min}$ equilibrium period. Minutes 6,7 and minutes 9,10 were used for analysis

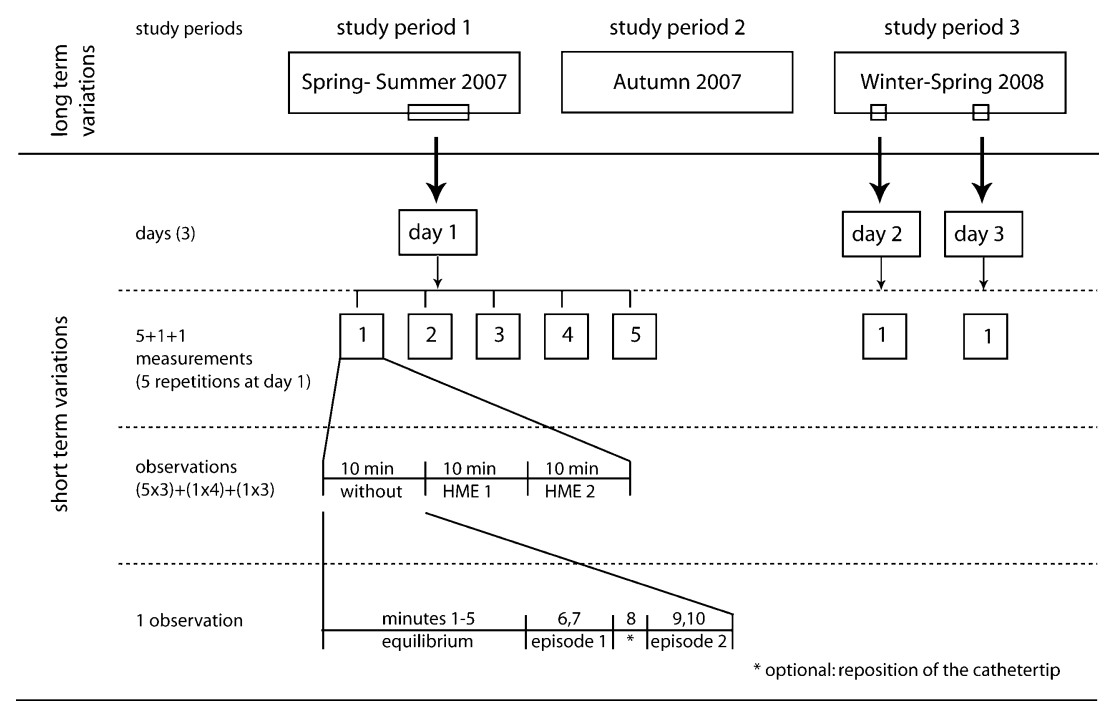


Hörby, Sweden) are given. The results for the other HMEs will be reported separately.

In previous studies, we found that the endotracheal temperature and humidity equilibrium was reached within 5 min [14]. Two 2 min episodes (minutes 6,7 and 9, 10) of each observation were used for analysis. The catheter tip was intentionally moved (vertically and horizontally) and repositioned as close as possible to the starting position within the tracheostoma during the eighth minute in some patients $(n=8)$. Since the HME device was not removed in such an instant, a new equilibrium period was not necessary.

Due to the HME effect of the tracheal mucosa, higher temperature and humidity values can be expected in measurements deeper inside the trachea (vertical movement) compared to measurements close to the tracheostoma opening. Therefore, the effect of vertical movement was also investigated in 11 patients, either before or just after the last 2 min episode (minutes 9, 10).

\subsection{Airway climate explorer (ACE)}

The ACE is purpose-built for the assessment of endotracheal temperature and humidity. The development and first validations have been described in detail previously [14]. In summary, a small diameter $(5 \mathrm{~mm})$ sample catheter is proximally connected to a sensor house in which a fast humidity sensor is built. Both the sample catheter and the sensor house are internally heated to $40^{\circ} \mathrm{C}$ in order to prevent condensation of water vapour within the sample catheter and/or sensor house. For the assessment of temperature a thermocouple (MLT1402 T-type Ultra Fast Thermocouple Probe (IT-23), response time $5 \mathrm{~ms}$, accuracy $\pm 0.1^{\circ} \mathrm{C}$; ADInstruments Ltd, Oxfordshire, UK) is placed just inside the distal tip of the central, air-sampling canal of the sample catheter. The airflow during respiration is sampled with a constant rate of $0.6 \mathrm{~L} / \mathrm{min}$.

\subsection{Breathing monitoring}

The breathing frequency was monitored with respiratory inductive plethysmography (Respitrace QDC, Viasys Healthcare, Houten, The Netherlands). In 13 patients, airflow was measured with a spirometer flowhead (flowhead MLT300L, Adinstruments, Oxfordshire, UK) placed on the peristomal adhesive (with or without HME device) by use of an airtight attachment with a cardboard tube during the two minute periods before and after each 10 min observation period.

\subsection{Room conditions}

Room conditions were monitored with a calibrated temperature and humidity sensor (Testo BV, Almere, The
Netherlands). The median room environment temperature was $23.7^{\circ} \mathrm{C}$ (range $22.6-27.8^{\circ} \mathrm{C}$, SD 1.0 ), the median room absolute humidity was $6.4 \mathrm{mg} \mathrm{H}_{2} \mathrm{O} / \mathrm{L}$ (range $5.9-11.1 \mathrm{mg}$ $\mathrm{H}_{2} \mathrm{O} / \mathrm{L}, \mathrm{SD}$ 1.6) and the median room relative humidity was $30.1 \%$ (range $22.6-57.7 \%$, SD 7.6).

\subsection{Data acquisition}

All signals are simultaneously recorded at a sample rate of 100/s with a multi channel data acquisition system (Powerlab) with additional software (Chart 5.4.1, Adinstruments Ltd, Oxfordshire, UK, Labview 8.2, National Instruments Netherlands BV, Woerden, The Netherlands).

\subsection{Sensors and calibrations}

Because the primary output of the temperature and humidity sensor is voltage $(V)$, both sensors must be calibrated at least once, either after replacement of the sensor or after transport of the ACE. The calibration process has been described previously [14]. All calibrations were grouped per thermocouple and per humidity sensor and the two best fitting data-points were used for the final calibration per sensor (see Fig. 2).

During the study period six different humidity sensors and two thermocouples were used. Previous experiments revealed that the accuracy of the humidity sensor (RS92K, Vaisala Oyj, Helsinki, Finland) can be reduced by two events [14]. These are (1) the aspiration of water or mucous into the system which can cause lengthening of the response time with leads to inaccurate results if the response time exceeds $0.7 \mathrm{~s}$ or even complete failure of the humidity sensor and (2) an insufficiently heated sample

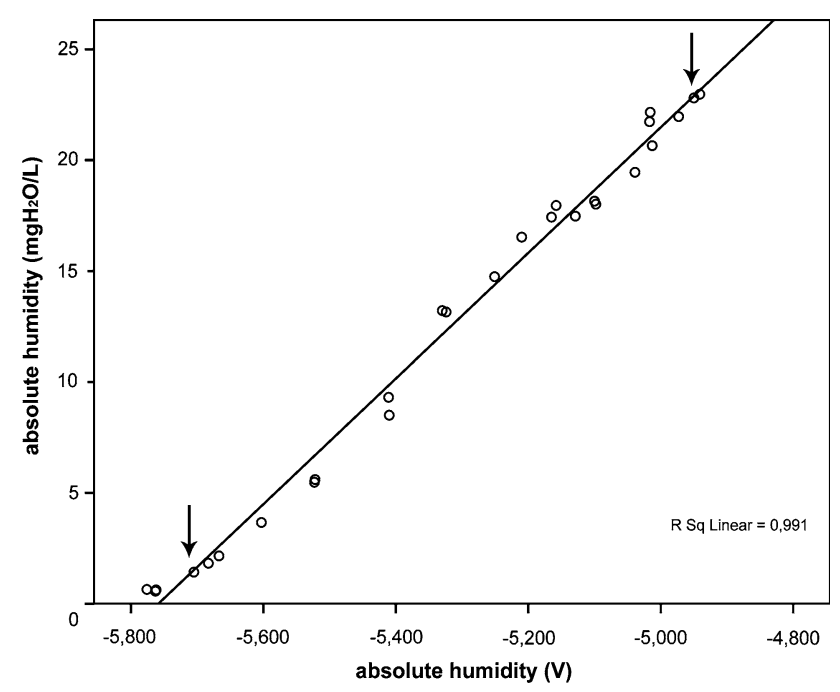

Fig. 2 Multiple calibrations of sensor 1. Two best fitting data-points (one low and one high; arrows) are used for the overall final calibration for this particular sensor 
catheter which will lead to condensation of water inside the canal of the sample catheter. Both conditions can be detected (1) by frequently checking the response time of the humidity sensor by using a two-stream system [14], and (2) by inspection of all raw data for a shift between temperature and humidity sensor which occurs in the event of condensation. Data measured with a humidity sensor with response times longer than $0.7 \mathrm{~s}$ were excluded from analysis. The median response time of the humidity sensor was $0.12 \mathrm{~s}$ (range 0.09-0.23 s) during expiration and $0.31 \mathrm{~s}$ (range $0.2-0.63 \mathrm{~s}$ ) during inspiration.

\subsection{Data processing}

Individual breaths from each measurement period were identified using a peak detection algorithm ('peaks'Splus). The time between two end-exhalations was defined as the full breath length (FBL), and the time between endexhalation and end-inhalation as the inhalation breath length (IBL). The difference between FBL and IBL is the exhalation breath length (EBL). Detection of the endexhalation en end-inhalation points was inaccurate. Therefore, the midpoints of the inhalation and exhalation periods were used to approximate the IBL, EBL and FBL (see Fig. 3). The end-inspiratory and end-expiratory values are referred to as minimums and maximums respectively throughout this article. "Breaths" that had a difference

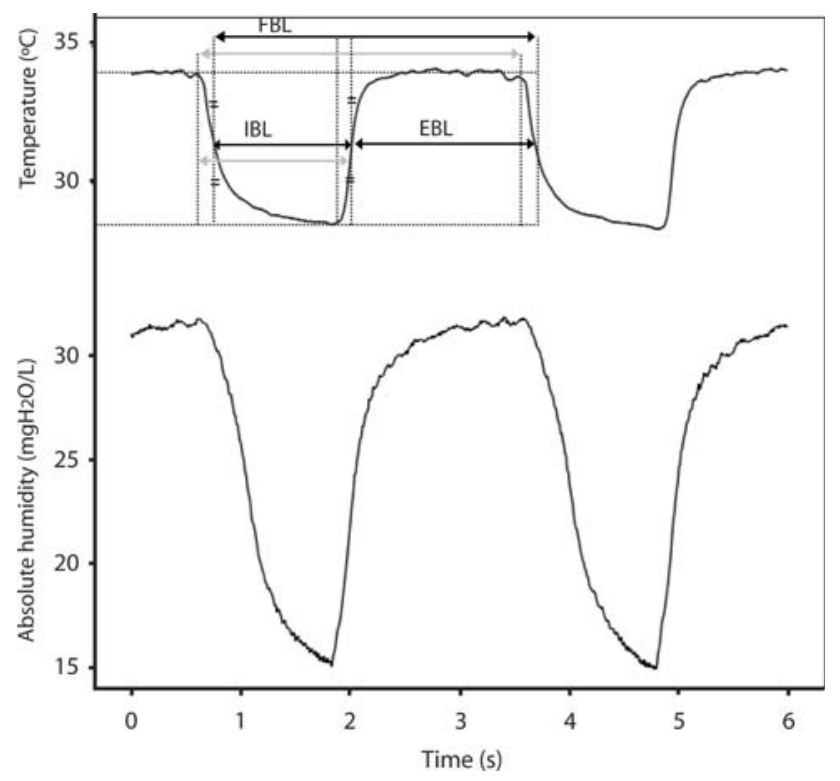

Fig. 3 The full breath length (FBL) is defined as the distance between two successive end-exhalations and the inhalation breath length (IBL) as the distance between the start of the inhalation and the start of exhalation (grey arrows). The IBL and FBL are approximated as the time between the midpoints of the inhalation and exhalation periods (black arrows). The EBL is the difference between IBL and FBL between the minimum and maximum humidity values of less than $2 \mathrm{mg} \mathrm{H}_{2} \mathrm{O} / \mathrm{L}$ were assumed to be cardiac oscillations [14] and therefore were excluded.

\subsection{Data analysis and modelling}

The analysis of temperature minima, temperature maxima, IBL, EBL and FBL was performed using five linear mixed effects models. In each model the variable of interest was associated with HME type. It was anticipated that room temperature would influence temperature minimums, but the range in room temperatures was too small for this association to be determined. Therefore, room temperature was not included in the model. No correlation between temperature minima and IBL was found. Humidity minima however, were positively linearly related to room humidity. Humidity minima were expected to depend exponentially on IBL due to the response time of the sensor which is not sufficiently short compared to the IBL. Therefore, a nonlinear exponential-decay mixed effects model was used to analyse both humidity minima and maxima simultaneously. This model estimated the mean initial humidity values ( $\mathrm{IBL}=0)$, the decay rate $(A 3)$ which represents the reaction time (in seconds) and the asymptotic humidity minima (A1) at infinitely long IBL. The asymptotic minima $A 1$ were related linearly to room humidity $\left(H_{\mathrm{r}}\right)$. The initial humidity values are equivalent to the humidity maxima (A2), as the start of inhalation equals the end of exhalation. Using the estimated IBL from the mixed effects model for IBL, the clinically observed humidity minima $\left(H_{\min }\right)$ can be calculated using the following equation:

$$
\begin{aligned}
H_{\min }= & A 1+\left(0.94 \times H_{\mathrm{r}}\right)+\left(A 2-A 1-\left(0.94 \times H_{\mathrm{r}}\right)\right) \\
& \times \exp (-\mathrm{IBL} / A 3)
\end{aligned}
$$

The equation represents the exponential decay of $H_{\min }$ as a function of IBL with reaction time $A 3$ from the maximum humidity $A 2$ towards the asymptotic minimum $A 1$ corrected for the room humidity $\left(H_{\mathrm{r}}\right) . A 1, A 2$, and IBL are all dependent on HME type and on patients, while the reaction time (A3) may depend only on HME type (and is fixed for all patients).

To account for correlations between observations taken at the same time (during one measurement session), during the same study period and from the same patient, observation identifier was nested within study period, which was nested within patient identifier as random variables. As no changes (linear or otherwise) were anticipated over time, both study period and observation identifier were implemented as categorical random variables. These three levels of nested random effects allow for the assessment of the variability due to inter-patient variations and long-term (in between study periods) and short-term (within a study period) intra-patient variations. To answer the question 
how movement and replacement of the catheter tip within an observation period affects the results, all five models were extended by allowing the standard deviations of the unexplained variation to vary depending whether the observations were from before or after the replacement.

The random effects structure for the non-linear humidity model was the same as that used in the temperature and breath length models, however only the initial (A2) and asymptotic $(A 1)$ parameters were associated with the random variables. Similarly the model extensions were implemented in the humidity analysis.

The difference between the estimate of clinical temperature and humidity minimums obtained with and without an HME was tested using $t$ tests using the estimates of the residual standard errors at the clinical minimums (differing by HME type) and the degrees of freedom estimate obtained if a standard linear mixed effects model was employed. The standard deviations of the different levels of random effects are used to indicate the different amounts of inter- and intra-patient variability. For the linear breath length and temperature models, 95\% CIs of the standard deviations are reported, for the non-linear humidity model a onefold cross validation (in which an entire patient is excluded each iteration) is used to provide the range of standard deviations.

The statistical analysis was conducted using Splus v6.2 pro.

\subsection{Raw data analysis}

In order to check whether the model estimates are accurate reflections of the measured values, the temperature and humidity values of the raw data were also read out by hand. In each observation, one representative breath with a clinical relevant (i.e. type specific) breath length was chosen within the last $2 \mathrm{~min}$ episode. The temperature and humidity minimum and maximum values and the corresponding IBL of this breath were registered in a database (Microsoft Office Excel v2003). The means of these values were compared with the estimates of the statistical model. Because the estimates of the humidity model are IBL dependent, the humidity model estimates were calculated for the breath-specific IBL, which was read out by hand.
Table 1 An overview of the number of days, measurements and observations per patient per study period which were used for breath length, temperature and humidity data analysis
Patient numbers 6, 9 and 12 were excluded from the final analysis and therefore not included in the table

\begin{tabular}{|c|c|c|c|c|}
\hline Study periods & $\begin{array}{l}\text { Patient } \\
\text { number }\end{array}$ & $\begin{array}{l}\text { Day per } \\
\text { study period }\end{array}$ & $\begin{array}{l}\text { Measurements } \\
\text { per day(s) }\end{array}$ & $\begin{array}{l}\text { Observations per } \\
\text { measurement(s) }\end{array}$ \\
\hline \multirow[t]{7}{*}{ Spring-Summer 2007} & 1 & 1 & 5 & $(3+3+3+3+3)$ \\
\hline & 2 & 1 & 2 & $(2+3)$ \\
\hline & 3 & 1 & 1 & 3 \\
\hline & 4 & 1 & 1 & 3 \\
\hline & 5 & 1 & 1 & 3 \\
\hline & 7 & 2 & $2 / 3$ & $(3+3) /(3+3+3)$ \\
\hline & 8 & 2 & $2 / 2$ & $(3+3) /(3+3)$ \\
\hline \multirow[t]{7}{*}{ Autumn 2007} & 5 & 1 & 1 & 3 \\
\hline & 10 & 1 & 1 & 3 \\
\hline & 11 & 1 & 1 & 3 \\
\hline & 13 & 1 & 1 & 3 \\
\hline & 14 & 1 & 1 & 3 \\
\hline & 15 & 1 & 1 & 3 \\
\hline & 16 & 1 & 1 & 3 \\
\hline \multirow[t]{11}{*}{ Winter-Spring 2008} & 1 & 2 & $1 / 1$ & $4 / 3$ \\
\hline & 2 & 3 & $1 / 1 / 1$ & $4 / 3 / 3$ \\
\hline & 3 & 1 & 1 & 4 \\
\hline & 4 & 3 & $1 / 1 / 1$ & $4 / 2 / 3$ \\
\hline & 5 & 1 & 1 & 3 \\
\hline & 7 & 3 & $1 / 1 / 1$ & $4 / 3 / 3$ \\
\hline & 8 & 2 & $1 / 1$ & $4 / 3$ \\
\hline & 11 & 2 & $1 / 1$ & $3 / 3$ \\
\hline & 13 & 1 & 1 & 3 \\
\hline & 14 & 1 & 1 & 3 \\
\hline & 15 & 2 & $1 / 1$ & $3 / 3$ \\
\hline 3 study periods & 13 patients & 37 days & 47 measurements & 145 observations \\
\hline
\end{tabular}




\section{Results}

In the first study period humidity measurements were more often invalid due to the operator learning curve who had to become experienced in the avoidance of mucous suction. After exclusion humidity data were available fore analysis in 13 patients (in total 47 measurements, 145 observations, and 8,043 breaths) Table 1 shows the details of the measurements per patient.

Temperature and breath length data were available for an additional three patients (total 16 patients, 76 measurements, 208 observations, and 11,824 breaths). The results of temperature and breath length analyses for both the 13 and 16 patient datasets were very similar. For consistency with the analysis of the humidity data, we report all results from the 13 patient data set.

\subsection{Breathing pattern}

Breathing with and without HME device resulted in almost identical full breath lengths (FBL) (median $3.6 \mathrm{~s}$, see Table 2). In contrast, median inhalation breath lengths (IBL) with an HME covering the stoma was significantly shorter than without HME (1.05 vs. $1.35 \mathrm{~s} ; P<0.001)$ and EBL was significantly longer with HME (2.69 vs. 2.19 s; $P<0.0001)$.

The median maximum spirometric airflow assessed before and after the ACE measurements was $0.5 \mathrm{~L} / \mathrm{s}$ and the median tidal volume was $0.47 \mathrm{~L}$.

\subsection{Raw data and model fit for humidity and temperature}

Minimum and maximum values of endotracheal temperature and humidity of all breaths of one measurement session from the one typical patient (patient 1 in Table 1) are shown in Fig. 4. In Fig. 4a the temperature values are plotted against IBL. The point where IBL equals zero represents the end of an exhalation and yields the average maximum temperature values with and without HME. This analysis shows that temperature minimums do not depend on IBL.

In Fig. 4b, the endotracheal absolute humidity values and the estimated fit of the relationship between absolute humidity and IBL are shown. The end of exhalation (i.e. the start of inspiration at $\mathrm{IBL}=0$ ) corresponds to the maximum humidity values (A2). During inhalation, the minimum humidity values are dependent on the IBL. The minimum humidity values decrease when the IBL is lengthened. If the duration of the inhalation would be sufficiently long, the humidity would reach an equilibrium minimum value represented by the asymptote $(A 1)$. In practice, the IBL is never long enough to reach this value. The minimum values that are attained decrease exponentially with the IBL. The decay of this curve is described by the reaction time $(A 3)$, which depends on HME type, but is a fixed value for all patients. With HME, the $A 3$ turns out to be longer $(0.80 \mathrm{~s})$ than without $\mathrm{HME}(0.51 \mathrm{~s}$; $P<0.001)$ : i.e. the curve without HME decreases faster than the curve with HME before the asymptotic minimal value is reached. The clinically relevant humidity minimums $\left(H_{\min }\right)$ can be determined from Eq. 1 .

\subsection{Temperature and humidity minimums and maximums}

The model estimates of the temperature minimums were $26.9^{\circ} \mathrm{C}$ with $\mathrm{HME}$ and $28.5^{\circ} \mathrm{C}$ without $\mathrm{HME}$ (difference $-1.6^{\circ} \mathrm{C} ; P<0$. 001). The model estimates of the clinically relevant humidity minimums $\left(H_{\min }\right.$ from Eq. 1 using the HME-dependent IBL) with and without HME were $22.8 \mathrm{mg} \mathrm{H}_{2} \mathrm{O}$ and $17.0 \mathrm{mg} \mathrm{H}_{2} \mathrm{O} / \mathrm{L}$, respectively
Table 2 The means of the IBL FBL and minimum and maximum temperature and humidity values are shown in this table

All data are model estimates. For the absolute humidity data, also the $A 1, A 2$ (maximum), $A 3$ (reaction time) and the clinical minimum humidity values at type specific IBL (1.06 and $1.35 \mathrm{~s}$, respectively with and without HME) and at the same IBL $(1.1 \mathrm{~s}$ for both with and without HME)

\begin{tabular}{lcccc}
\hline & Without HME & With normal HME & Difference & $P$ value \\
\hline Breaths (s) & & & & \\
Inhalation breath length (IBL) & 1.35 & 1.05 & -0.30 & $<0.0001$ \\
Exhalation breath length (EBL) & 2.19 & 2.61 & +0.42 & $<0.0001$ \\
Full breath length (FBL) & 3.55 & 3.65 & +0.10 & $\mathrm{NS}$ \\
Temperature ( ${ }^{\circ} \mathrm{C}$ ) & & & & \\
Minimum & 28.5 & 26.9 & -1.60 & $<0.001$ \\
Maximum & 34.4 & 34.5 & +0.10 & $\mathrm{NS}$ \\
Absolute humidity (mgH & & & \\
A1 & & & +2.9 & $<0.0001$ \\
Minimum (type specific IBL) & 17.0 & 12.6 & +5.8 & $<0.0001$ \\
Minimum (IBL $=1.1 \mathrm{~s}$ ) & 17.7 & 22.8 & +4.9 & $<0.0001$ \\
$A 2$ (maximum) & 33.5 & 22.6 & +0.7 & $<0.005$ \\
$A 3$ (reaction time) in seconds & 0.51 & 34.2 & +0.29 & $<0.001$ \\
\hline
\end{tabular}



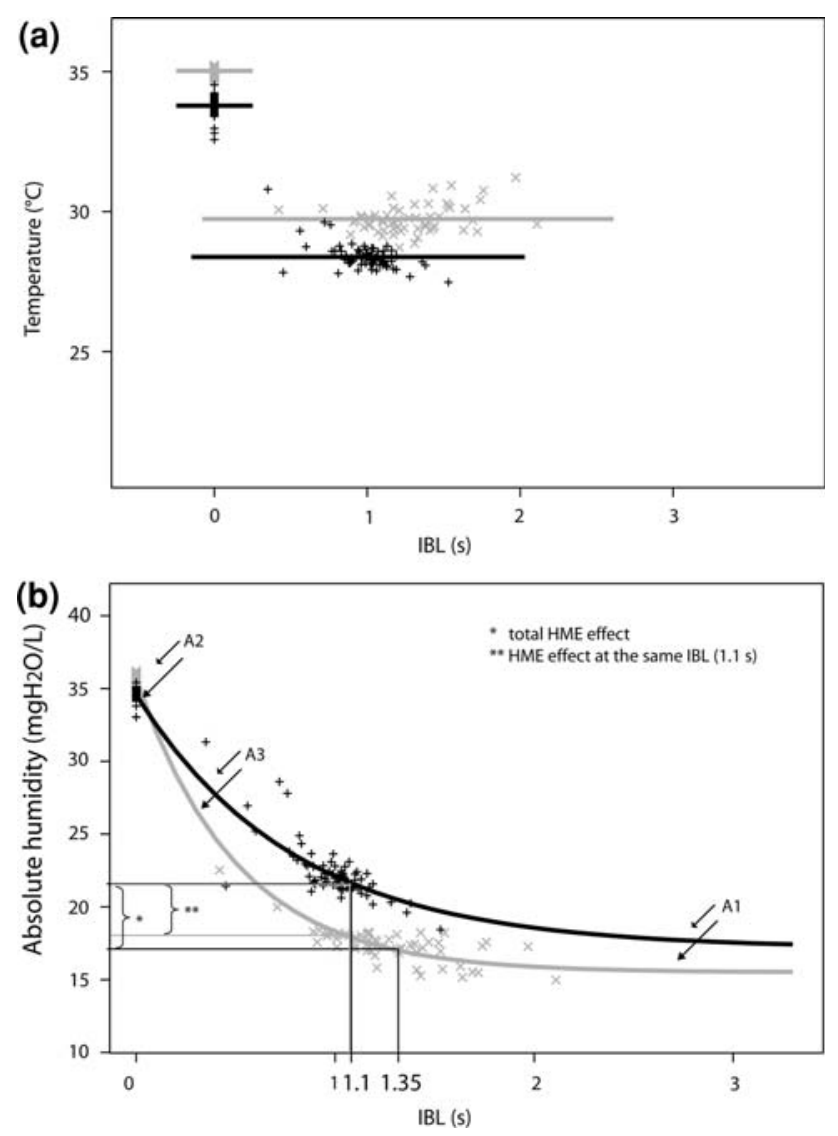

Fig. 4 Temperature and humidity data from the first measurement of patient 1 are given as an example. Temperature and humidity values are plotted against inhalation breath length (IBL). The point IBL equals zero represents the end of an exhalation and yields the average maximum temperature and humidity values with and without HME. All lines and curves are model fits. (black, $+=$ normal HME; gray, $x=$ without HME); a minimum and maximum temperature values. b Minimum and maximum humidity values. $A 1$ is the asymptote representing estimated minimum humidity values if IBL goes to infinity, $A 2$ the mean of the maximum humidity values and $A 3$ the reaction time. In this measurement: $A 1=11.1 \mathrm{mg} \mathrm{H}_{2} \mathrm{O} / \mathrm{L}$ and $A 2=$ $34.6 \mathrm{mg} \mathrm{H}_{2} \mathrm{O} / \mathrm{L}$ (with HME); $A 1=9.4 \mathrm{mg} \mathrm{H}_{2} \mathrm{O} / \mathrm{L}$ and $A 2=$ $35.7 \mathrm{mg} \mathrm{H}_{2} \mathrm{O} / \mathrm{L}$ (without $\mathrm{HME}$ ). Room humidity $\left(H_{\mathrm{r}}\right)=6.4 \mathrm{mg}$ $\mathrm{H}_{2} \mathrm{O} / \mathrm{L}$

$(P<0.001)$. The clinically relevant $\mathrm{HME}$ effect was therefore $5.8 \mathrm{mg} \mathrm{H}_{2} \mathrm{O} / \mathrm{L}$. This difference was $4.9 \mathrm{mg} \mathrm{H}_{2} \mathrm{O}$ / $\mathrm{L}$ if both minimum humidity values were calculated at the same IBL (1.1 s). Table 2 provides an overview of the results of the means of all output variables.

\subsection{Raw data analysis and check of the model estimates}

The means of the minimum and maximum temperature values of the raw data analysis differed on average with 0.14 and $0.10^{\circ} \mathrm{C}$, respectively, from the corresponding model estimates. The means of the minimum and maximum humidity values of the raw data analysis differed on average with $0.24 \mathrm{mg} \mathrm{H}_{2} \mathrm{O}$ and $0.08 \mathrm{mg} \mathrm{H}_{2} \mathrm{O} / \mathrm{L}$, respectively, from the model estimates calculated for the same (breath specific) IBL. Because these differences are quite small, we can assume that the model estimates are accurate reflections of the measured values.

\subsection{Inter and intra patient variability}

Table 3 gives an overview of the inter- and intra-patient variability including the $95 \%$ confidence intervals. The intra-patient variability was divided in short-term variations (within study periods: observations were repeated either within the same day or on different days) and longterm variations (in between study periods). The short- and long-term variations had a similar magnitude in all parameters.

For the temperature and humidity minimums, the interpatient variability was much smaller than the short- and long-term intra-patient variations. For EBL and FBL, the opposite was the case. The inter-patient variability of IBL and temperature maximums was similar to the intra-patient variations.

\subsection{Repositioning of the catheter tip}

When the catheter tip was moved within the tracheostoma (in the 8th minute of the observation period) and repositioned to its starting position, the variations between the observations before and after the repositioning were not significantly different. After repositioning the catheter tip from "standard" stoma depth $(1 \mathrm{~cm})$ upward close to the tracheostoma opening, a decrease in both temperature and humidity minimums was observed (respectively $0.45^{\circ} \mathrm{C}$ and $0.83 \mathrm{mg} \mathrm{H}_{2} \mathrm{O} / \mathrm{L}$, determined at the clinically relevant IBL). For the maximums, almost no difference was found (decrease $<0.2^{\circ} \mathrm{C}$ and $<0.1 \mathrm{mg} \mathrm{H}_{2} \mathrm{O} / \mathrm{L}$ ).

\section{Discussion and conclusion}

\subsection{Intra- and inter-patient variability}

In this study, we determined the variations in endotracheal temperature and humidity values, which have to be taken in account when measuring the in vivo climate changes in the trachea in laryngectomized individuals. Intra-patient variability contributes most to these variations, whereas the inter-patient variability is much smaller. The intra-patient variability is particularly large for the minimum humidity values for both short-term $\left(2.04 \mathrm{mg} \mathrm{H}_{2} \mathrm{O} / \mathrm{L}\right)$ and long-term (1.60 $\mathrm{mg} \mathrm{H}_{2} \mathrm{O} / \mathrm{L}$ ) variations. These intra-patient variations can neither be caused by the inhalation breath length (IBL) nor by variations in the room humidity, because both IBL 
Table 3 The standard deviations of the intra- and inter-patient variation

\begin{tabular}{|c|c|c|c|}
\hline \multirow[t]{2}{*}{ Variability ( $n=8,043$ breaths) } & \multicolumn{2}{|l|}{ Intra patient } & \multirow[t]{2}{*}{ Inter patient } \\
\hline & Short term variability & Long term variability & \\
\hline \multicolumn{4}{|l|}{ Breath length (s) } \\
\hline Inhalation breath length (IBL) & $0.11(0.10-0.13)$ & $0.08(0.04-0.16)$ & $0.12(0.07-0.21)$ \\
\hline Exhalation breath length (EBL) & $0.32(0.27-0.36)$ & $0.25(0.14-0.46)$ & $0.52(0.33-0.82)$ \\
\hline Full breath length (BL) & $0.33(0.28-0.38)$ & $0.36(0.21-0.60)$ & $0.60(0.37-0.98)$ \\
\hline \multicolumn{4}{|l|}{ Temperature $\left({ }^{\circ} \mathrm{C}\right)$} \\
\hline Minima & $0.73(0.63-0.83)$ & $1.06(0.77-1.45)$ & $0.04(0.03-0.08)$ \\
\hline Maxima & $0.36(0.32-0.41)$ & $0.45(0.28-0.72)$ & $0.37(0.16-0.85)$ \\
\hline \multicolumn{4}{|l|}{ Absolute humidity (mg $\left.\mathrm{H}_{2} \mathrm{O} / \mathrm{L}\right)$} \\
\hline$A 1$ (asymptotic minima) & $2.04(1.7-2.09)$ & $1.60(1.2-1.7)$ & $0.39(0.14-0.57)$ \\
\hline A2 (maxima) & $1.04(0.7-1.1)$ & $1.55(1.4-1.6)$ & $0.35(0.06-0.54)$ \\
\hline
\end{tabular}

The short-term variability is the variability within study periods and long-term variability is that in between study periods. For breath length and temperature the $95 \%$ confidence intervals are reported. For humidity the range of a 1-fold cross validation is reported

and room humidity were part of the model. The most likely major cause of these variations is inconsistency in the positioning of the catheter tip in the trachea at the beginning of each new observation.

During each observation, endotracheal temperature and humidity should be measured at $1 \mathrm{~cm}$ stoma depth according to the protocol, but in practice, it appeared to be rather difficult to position the catheter tip at exactly the same position in the tracheostoma. In the presence of an HME, correct positioning of the catheter tip is even more difficult as visual inspection of the catheter tip is impossible. Additionally, the position of the catheter tip may have unintentionally changed during some observations, when a patient had difficulties sitting in the same position all the time.

Previously we have investigated the impact of horizontal variations in the positioning and found that the temperature and humidity minimums in the centre of the trachea are about $1{ }^{\circ} \mathrm{C}$ and $2 \mathrm{mg} \mathrm{H}_{2} \mathrm{O} / \mathrm{L}$, respectively lower than close to the tracheal wall, indicating that the flow is close to turbulent [14]. However, this conclusion was based on the data of only four patients (only 2 with an HME in situ) of which one patient had considerably larger deviations. Positioning errors probably have a larger impact on the intra-patient variability, because not only horizontal positioning, also vertical positioning is important as a sharp humidity gradient in the trachea from the stoma down to the peripheral pathways exists during inhalation. Dry air (about $6 \mathrm{mg} \mathrm{H}_{2} \mathrm{O} / \mathrm{L}$ ) enters the trachea and is subsequently conditioned by the tracheal and pulmonary mucosa (which act as an HME), until the high humidity of the peripheral pulmonary airways ( $44 \mathrm{mg} \mathrm{H}_{2} \mathrm{O} / \mathrm{L}$ ) is reached. This point is called the Isothermal Saturation Boundary (ISB) where inhaled air has reached body temperature $\left(37^{\circ} \mathrm{C}\right)$ and is $100 \%$ saturated with water vapour $[3,10,14]$. The measurements close to the tracheostoma opening (or directly behind the HME) confirm the existence of the sharp humidity gradient, since the minimum humidity values close the tracheostoma opening are almost $1 \mathrm{mg} \mathrm{H}_{2} \mathrm{O} / \mathrm{L}$ lower than at $1 \mathrm{~cm}$ stoma depth. This relatively large gradient at such a small difference in stoma depth implicates that a precise vertical positioning is important, not only for humidity but also for temperature minimum (end-inspiratory) values. The temperature gradient however is less "steep" $\left(12^{\circ} \mathrm{C}\right.$ vs. $\left.28 \mathrm{mg} \mathrm{H}_{2} \mathrm{O} / \mathrm{L}\right)$ as inhaled air at room temperature $\left(23^{\circ} \mathrm{C}\right)$ is in the trachea warmed up to $37^{\circ} \mathrm{C}$ at ISB. This is in agreement with our measurements close to the tracheostoma and at $1 \mathrm{~cm}$ stoma depth (difference $0.45^{\circ} \mathrm{C}$ versus $0.83 \mathrm{mg} \mathrm{H}_{2} \mathrm{O} / \mathrm{L}$ ) and with the smaller intra-patient variations in temperature (about $1^{\circ} \mathrm{C}$ versus about $2 \mathrm{mg} \mathrm{H} \mathrm{H}_{2} \mathrm{O} / \mathrm{L}$ ).

During exhalation, however, hardly any gradient exists in the trachea neither in the horizontal [14], nor in the vertical direction. Indeed the intra-patient variations in the temperature maximums are small. The larger variations in the humidity maximums probably have a different cause. The endotracheal humidity maximum values are about $20 \mathrm{mg} \mathrm{H}_{2} \mathrm{O} / \mathrm{L}$ higher than the maximum humidity attained during calibration of the humidity sensor at room temperature [12]. Therefore, the calibration curve has to be extrapolated and is, consequently, less accurate in measuring these high endotracheal humidity values. In particular when different sensors were used in different study groups (long term), this error obviously contributes to the long-term variations.

Long-term effects in both maximums and minimums may also be due to patient related changes such as the influence of environmental factors on the tracheal mucosa. For instance, dry air and/or excessive water loss through the respiratory tract will lead to increased viscosity of the 
mucous layer in the tracheobronchial tree and will reduce the overall moistening effect of the peripheral pulmonary pathways [9]. In the end, after repeated inspiration of cold and dry air, the ISB shifts up towards the peripheral airways $[3,10,14]$. This leads to a longer lasting colder tracheal mucosa, which reasonably contributes to differences in end-expiratory values.

\subsection{Breath length}

Although the inter-patient variations of temperature and humidity values are quite small, the variations in FBL and EBL parameters are primarily patient dependent. Whether this is caused by neurological or pulmonary stimulants or by the physical condition of the patient at that moment, is not known [2]. Breathing with HME does not influence the average breathing frequency (or FBL), but causes a significantly shorter IBL (1.05 s) compared to breathing without HME (1.35 s). Although most HME devices (including the normal HME used in this study) only partially compensate the breathing resistance of the normal upper airways [13], a large difference in breathing resistance still exists between breathing with and breathing without HME. Probably, the increased breathing resistance during breathing with HME underlies the shorter IBL (and longer EBL).

\subsection{HME effect}

Breathing through an HME increases endotracheal minimum and maximum humidity values. The minimum humidity values are dependent on IBL, since the reaction time $A 3$ of the humidity measurements is too long too follow the whole breathing curve at very short IBLs. Minimally the reaction time $A 3$ will be equal to the response time of the humidity sensor, as the delay of the sensor is always present. The reaction time $A 3$ during humidity measurements without HME is about $0.5 \mathrm{~s}$, which is about equal to the response time of the humidity sensor. With HME the reaction time $A 3$ is about $0.3 \mathrm{~s}$ longer than the response time of the humidity sensor. The additional reaction time probably represents the gradual evaporation of water from the HME so that the air in the trachea stays longer humid than without HME.

The moistening effect of the HME is enhanced by the shorter IBL when breathing with HME. In designing HMEs it is important to understand the moistening capacities of both contributors (water evaporation by the HME foam and the impact of IBL differences) separately. To be informed about the contribution of the HME foam only, the difference with and without HME must be calculated at the same IBL to exclude the enhanced HME effect generated by the shortening of the IBL. Taking the effect of shortened IBL into account the clinical HME effect is $5.8 \mathrm{mg} \mathrm{H}_{2} \mathrm{O} / \mathrm{L}$. The net HME effect at the IBL of $1.1 \mathrm{~s}$ (which we used in our previous study [14]) is $4.9 \mathrm{mg} \mathrm{H}_{2} \mathrm{O} / \mathrm{L}$. In this previous study, based on only six patients [14] we found a net HME effect of $3.2 \mathrm{mg} \mathrm{H}_{2} \mathrm{O} / \mathrm{L}$ without significant differences in IBL with and without HME. The difference may be due to the larger number of patients, but as this study has shown that intra-patient variation is quite large and probably due to positioning errors, the difference might also be the result of slight differences in insertion technique as different investigators (this study RS, previous study KZ [14]) performed the measurements.

\subsection{Limitations of the study}

The model used for the analysis of humidity is necessarily a simplification of a complex reality. The results for the variations are valid for the asymptotes $(A 1)$, which are the hypothetic minimum humidity values if the IBL goes to infinity. In other words, the variations are calculated at the end of extrapolated lines of the humidity values at clinical relevant IBL. Therefore, the absolute magnitude of the variations will not be entirely representative for the absolute magnitude of the humidity values at the clinically relevant IBL and the variability in this study may therefore rather be an overestimation. However, the relative contributions of the variations (e.g. short- vs. long-term variations) have given us insight in the meaning of the HME effects we observe.

Cardiac oscillations will influence the recorded temperature and humidity values [14]. Cardiac oscillations tend to occur at end of an inhalation and are then not recognised as such by the computer algorithm. Visual inspection of all data showed that the impact on the average temperature minimums is less than $0.1 \mathrm{mg} \mathrm{H}_{2} \mathrm{O} / \mathrm{L}$ and less than $0.1^{\circ} \mathrm{C}$.

\subsection{Conclusions}

When measuring the endotracheal temperature and humidity in laryngectomized patients the inter-patient variation is much smaller than the intra-patient variation. Consequently, only a limited number of laryngectomized patients has to be included in future studies of the endotracheal climate and/or the HME effect if a sufficient number of repetitions are accomplished, or stated otherwise, more repetitions in a few patients are just as useful as a few repetitions in many patients.

The vertical temperature and humidity gradient in the trachea during inspiration (and in some patients the flow turbulence) combined with positioning inaccuracies leads to significant intra-patient variations. This variation will hamper the comparison of studies of results for different HME's, in particular if they are performed by different 
protocols and/or observers. It is important that the design of future studies minimizes this variation. We have shown that it should be possible that the easier task of repositioning the catheter tip after intentional movement without removing it from the trachea did not lead to a significant contribution to the variations.

An HME not only leads to increased humidity minimums by the water preserving capacities of the foam, but also to a shortened IBL, which in turn enhances the HME effect. These two different contributions to the HME effect are important to understand when designing HMEs as they may be optimized separately (e.g. water retention capacities leading to a longer reaction time; the breathing resistance leading to a shortened IBL).

Acknowledgments This study was conducted under an unrestricted research grant of Atos Medical AB, Hörby, Sweden. Marcel van der Horst and Morgan Shehata, formerly Department of Medical Technology Development of the AMC, University of Amsterdam, are acknowledged for their continued technical support.

Open Access This article is distributed under the terms of the Creative Commons Attribution Noncommercial License which permits any noncommercial use, distribution, and reproduction in any medium, provided the original author(s) and source are credited.

\section{References}

1. Ackerstaff AH, Fuller D, Irvin M, Maccracken E, Gaziano J, Stachowiak L (2003) Multicenter study assessing effects of heat and moisture exchanger use on respiratory symptoms and voice quality in laryngectomized individuals. Otolaryngol Head Neck Surg 129:705-712. doi:10.1016/S0194-5998(03)01595-X

2. Benchetrit G (2000) Breathing pattern in humans: diversity and individuality. Respir Physiol 122:123-129. doi:10.1016/S00345687(00)00154-7

3. Branson RD (1999) Humidification for patients with artificial airways. Respir Care 44:630-641
4. Hilgers FJ, Ackerstaff AH, Aaronson NK, Schouwenburg PF, Van Zandwijk N (1990) Physical and psychosocial consequences of total laryngectomy. Clin Otolaryngol 15:421-425. doi: 10.1111/j.1365-2273.1990.tb00494.x

5. Jones AS, Young PE, Hanafi ZB, Makura ZG, Fenton JE, Hughes JP (2003) A study of the effect of a resistive heat moisture exchanger (trachinaze) on pulmonary function and blood gas tensions in patients who have undergone a laryngectomy: A randomized control trial of 50 patients studied over a 6-month period. Head Neck 25:361-367. doi:10.1002/hed.10264

6. Keck T, Durr J, Leiacker R, Rettinger G, Rozsasi A (2005) Tracheal climate in laryngectomees after use of a heat and moisture exchanger. Laryngoscope 115:534-537. doi: 10.1097/01.MLG.0000150417.51835.4F

7. Liener K, Durr J, Leiacker R, Rozsasi A, Keck T (2006) Measurement of tracheal humidity and temperature. Respiration 73:324-328. doi:10.1159/000088659

8. McRae RD, Jones AS, Young P, Hamilton J (1995) Resistance, humidity and temperature of the tracheal airway. Clin Otolaryngol 20:355-356. doi:10.1111/j.1365-2273.1995.tb00059.x

9. Mercke U, Toremalm NG (1976) Air humidity and mucociliary activity. Ann Otol Rhinol Laryngol 85:32-37

10. Rathgeber J, Zuchner K (1999) Foundations of artificial respiration. Manual for medical docters and nurses. Aktiv Druck \& Verlag GmbH, Ebelsbach

11. Rozsasi A, Leiacker R, Fischer Y, Keck T (2006) Influence of passive humidification on respiratory heat loss in tracheotomized patients. Head Neck 28:609-613. doi:10.1002/hed.20376

12. Zuur JK, Muller SH, de Jongh FH, van der Horst MJ, Shehata M, van Leeuwen J, Sinaasappel M, Hilgers FJ (2007) A newly developed tool for intra-tracheal temperature and humidity assessment in laryngectomized individuals: the airway climate explorer (ACE). Med Biol Eng Comput 45:737-745. doi: 10.1007/s11517-007-0217-7

13. Zuur JK, Muller SH, Sinaasappel M, Hart GA, Van Zandwijk N, Hilgers FJ (2007) Influence of heat and moisture exchanger respiratory load on transcutaneous oxygenation in laryngectomized individuals: a randomized crossover study. Head Neck 29:1102-1110. doi:10.1002/hed.20643

14. Zuur JK, Muller SH, Vincent A, Sinaasappel M, de Jongh FH, Hilgers FJ (2008) Assessment of tracheal temperature and humidity in laryngectomized individuals and the influence of a heat and moisture exchanger on tracheal climate. Head Neck 30:1072-1082. doi:10.1002/hed.20835 